
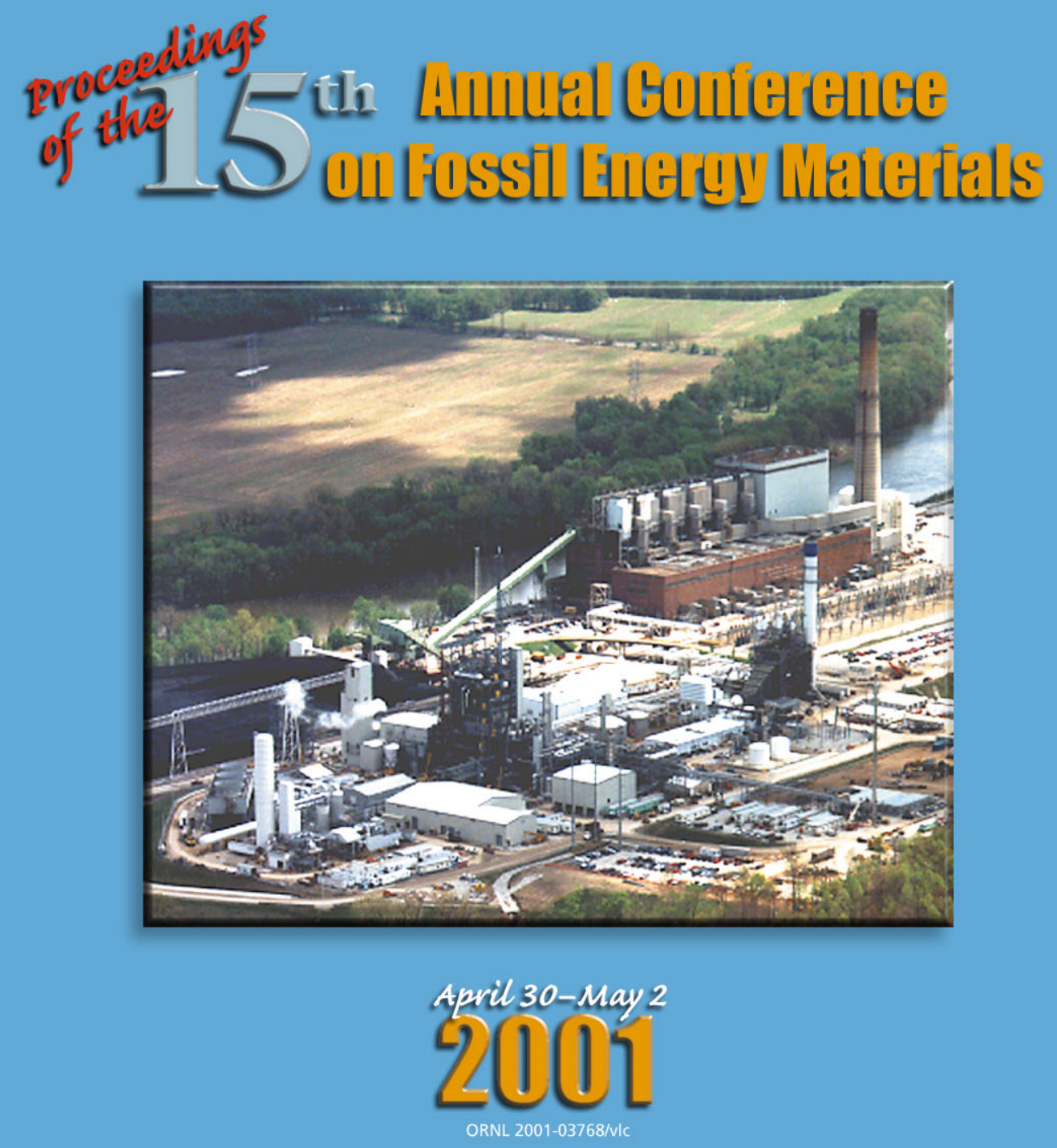

Published by:

Oak Ridge National Laboratory

Fossil Energy Program

Oak Ridge, TN 37831-6084
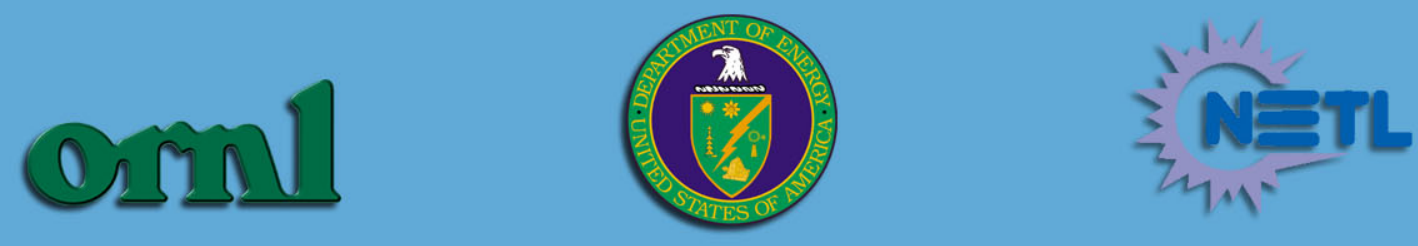


\section{DISCLAIMER}

This report was prepared as an account of work sponsored by an agency of the United States government. Neither the United States Government nor any agency thereof, nor any of their employees, makes any warranty, expressed or implied, or assumes any legal liability or responsibility for the accuracy, completeness, or usefulness of any information, apparatus, product, or process disclosed, or represents that its use would not infringe privately owned rights. Reference herein to any specific commercial product, process, or service by trade name, trademark, manufacturer, or otherwise does not necessarily constitute or imply its endorsement, recommendation, or favoring by the United States Government or any agency thereof. The views and opinions of authors expressed herein do not necessarily state or reflect those of the United States Government or any agency thereof.

Available to DOE and DOE contractors from the Office of Scientific and Technical Information, P.O. Box 62, Oak Ridge, TN 37831; prices available at 865-576-8401.

Available to the public from the National Technical Information Service, U.S. Department of Commerce, 5285 Port Royal Road, Springfield, VA 22161; prices available at 703-487-4650. 


\section{PREFACE}

The Fifteenth Annual Conference on Fossil Energy Materials was held in Knoxville, Tennessee, on April 30 through May 2, 2001. The meeting was sponsored by the U.S. Department of Energy's (DOE) Office of Fossil Energy through the Advanced Research Materials Program (ARM). The objective of the ARM Program is to conduct research and development on materials for longer-term fossil energy applications, as well as for generic needs of various fossil fuel technologies. The management of the program has been decentralized to the DOE Oak Ridge Operations Office and Oak Ridge National Laboratory (ORNL). The research is performed by staff members at ORNL and by researchers at other national laboratories, universities, and in private industry. The work is divided into the following categories: (1) structural, ceramics, (2) new alloys and coatings, (3) functional materials, and (4) technology development and transfer.

These proceedings were produced primarily from electronic files provided by the authors. They have been neither refereed nor extensively edited. However, most of the papers have already undergone technical review within the individual organizations before submission to the Program Office. The proceedings are available on the Fossil Energy home page at http://www.ornl.gov/fossil (Workshops and Conferences).

The successful completion of the conference and publication of the proceedings has required help from several people. The organizers wish to thank Angela Beach of the ORNL Conference Office for her help in the many arrangements, and the numerous staff and support personnel associated with the conference. Finally, we express our sincere appreciation to the authors whose efforts are the very basis of the conference.

\section{Conference Chairmen}

\section{ORNL}

R. R. Judkins

D. P. Stinton

R. W. Swindeman

P. F. Tortorelli

I. G. Wright

Albany Research Center

R. P. Walters 


\title{
PROCEEDINGS OF THE FIFTEENTH ANNUAL CONFERENCE ON FOSSIL ENERGY MATERIALS
}

April 30-May 2, 2001

Knoxville, Tennessee

\author{
R. R. Judkins
}

Fossil Energy Program Director

Date Published: September 2001

\author{
Prepared by \\ OAK RIDGE NATIONAL LABORATORY \\ P.O. Box 2008 \\ Oak Ridge, Tennessee 37831-6285 \\ managed by \\ UT-Battelle, LLC \\ for the \\ U.S. DEPARTMENT OF ENERGY \\ under contract DE-AC05-00OR22725
}
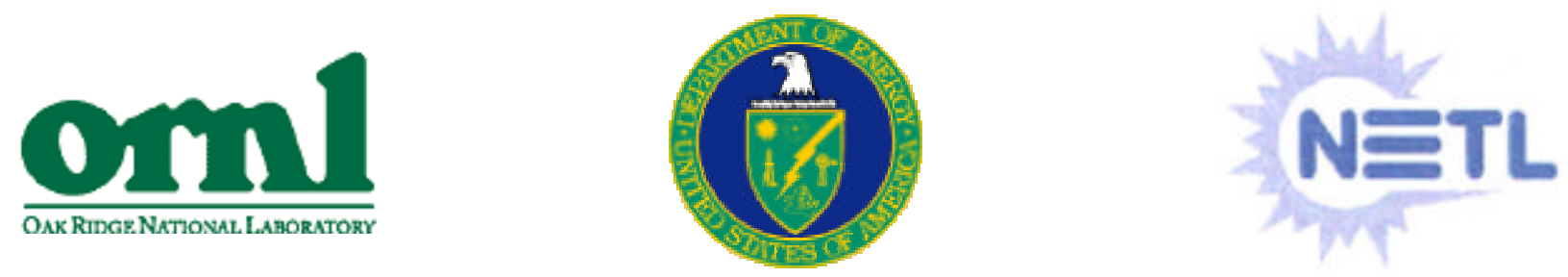


\section{CONTENTS}

\section{Disclaimer}

Preface

Final Program

\section{List of Attendees}

\section{Session I-Functional Materials}

Palladium Alloy Composite Membranes for Hydrogen Separation, S. N. Paglieri and S. A. Birdsell

Development of New Hybrid Organic-Inorganic and Inorganic Materials for Membranes at Sandia National Laboratories, D. A. Loy, C. J. Cornelius, J. T. Lean, and D. A. Schneider

Ceramic Membranes for High Temperature Hydrogen Separation, D. E. Fain, B. B. Marshall, T. Raj, K. D. Adcock, and L. E. Powell

Bismuth Oxide Solid Electrolyte Oxygen Separation Membranes, S. D. Nunn and E. A. Payzant

Solid Oxide Electrolyte Systems, L. R. Pederson, Y-S. Chou, G. W. Coffey, J. S. Hardy, K. J. Kerstetter, P. C. Rieke, and E. C. Thompson

Development of Novel Activated Carbon Composites, T. D. Burchell, R. Judkins, A. Gabbard, O. Omatete, and M. Rogers

\section{Session II-Coatings and Protection of Materials}

Sulfidation Resistance of Fe-Al Based Alloys with $\mathrm{Cr}$ and Ti Additions, J. R. Regina, J. N. DuPont, and A. R. Marder

Microstructure and Properties of $\mathrm{Fe}_{3} \mathrm{Al}$ Coatings, T. C. Totemeier, R. N. Wright, and W. D. Swank

Evaluation of Iron-Aluminide CVD Coatings for High Temperature Corrosion Protection, B. A. Pint Y. Zhang, P. F. Tortorelli, J. A. Haynes, and I. G. Wright

Development of Nondestructive Evaluation Technology for High-Temperature Coatings, W. A. Ellingson, C. Deemer, A. Parikh, and S. Erdmann

Atmospheric Pressure CVD of YSZ Using Stagnation Flow for Thermal Barrier Coatings, T. M. Besmann, J. C. McLaughlin, V. Varanasi, T. J. Anderson, and T. L. Starr

High-Temperature Materials Testing in a Pilot-Scale Coal Combustion System, J. Hurley and C. R. Crocker 


\section{Session III-New Alloys}

Progress on Developing Codes and Standards Requirements for New Alloys, R. W. Swindeman, M. L. Santella, and P. J. Maziasz

Oxide Dispersion-Strengthened Alloy Development, I. G. Wright, C. G. McKamey, and B. A. Pint Defect Reduction in $\mathrm{ODS}-\mathrm{Fe}_{3} \mathrm{Al}$, J. Ritherdon and A. R. Jones

High Temperature Performance of ODS Fe ${ }_{3} A l$-Based Alloy Tubes, B. K. Kad, C. G. McKamey, V. K. Sikka, and I. G. Wright

Corrosion Performance of Structural Alloys and Coatings in the Presence of Deposits, K. Natesan and C. Kraus

Development of Modified Alloy 803 with Improved Creep-Resistance above $800^{\circ} \mathrm{C}$, P. J. Maziasz, R. W. Swindeman, M. A. Harper, and G. D. Smith

\section{Session IV_Albany Research Center-Advanced Metallurgical Processes Program}

The Effect of Silicon and Aluminum Additions on the Oxidation Resistance of Lean Chromium Stainless Steels, J. S. Dunning, D. E. Alman, and J. C. Rawers

Oxidation in a Temperature Gradient, G. R. Holcomb, B. S. Covino, Jr., and J. H. Russell

Erosive Wear of Selected Materials for Fossil Energy Applications, T. A. Adler, J. C. Rawers, J. H. Tylczak, and J. A. Hawk

Advanced Titanium Processing, A. D. Hartman, K. K. Schrems, G. R. Holcomb, E. R. Argetsinger, J. S. Hansen, J. I. Paige, and P. C. Turner

Microchannel Devices, R. D. Wilson and D. E. Alman

Refractory Failure in IGCC Fossil Fuel Power Systems, C. P. Doğan, K-S. Kwong, J. P. Bennett, and R. E. Chinn

\section{Session V-Ultrahigh Performance Materials}

Multi-Phase Cr-Based Alloys for Aggressive High Temperature Environments, M. P. Brady, C. T. Liu, I. M. Anderson, P. F. Tortorelli, J. H. Zhu, I. G. Wright, V. K. Sikka, C. A. Walls, L. R. Walker, S. B. Waters, J. L. Wright, and C. A. Carmichael

Oxidation Resistance, Fracture Toughness and Creep Strength of Mo-Si-B Alloys Containing $\alpha-M o$, J. H. Schneibel and D. S. Easton

Novel Processing of Mo-Si-B Intermetallics for Improved Efficiency of Power Systems, M. J. Kramer, O. Degirmen, A. J. Thom, and M. Akinc 
Effect of Water Vapor on the Oxidation Behavior of Mo-Si-B Intermetallic Composites Near $1100^{\circ} \mathrm{C}$, M. J. Kramer, O. Degirmen, A. J. Thom, and M. Akinc

Development of a Commercial Process for the Production of Silicon Carbide Fibrils, R. D. Nixdorf, and H. Kimrey

\section{Posters}

\section{Fuel Cells}

Fabrication Technologies for Fuel Cell Applications, M. L. Santella, R. R. Judkins, and V. K. Sikka

High Density Infrared Sintering Process, C. A. Blue, T. R. Armstrong, B. L. Armstrong, C. Vodraska, M. E. Forrest, P. G. Engleman, and D. C. Harper

Modeling Long-Term Performance of Solid Oxide Fuel Cells: A Phenomenological Approach, X. Huang and K. Reifsnider

\section{Gas Cleaning/Separation}

Bismuth Oxide-Based Solid Electrolyte Systems, G. W. Coffey, L. R. Pederson, P. C. Rieke, and E. C. Thompson

\section{Gas Cleaning}

Progress in Development of High Temperature Metallic Filters for Advanced Fossil Energy Systems, I. E. Anderson, R. L. Terpstra, and B. Gleeson

\section{Coatings and Protection of Materials}

The Influence of Boron and Silicon in Improving the Oxidation Resistance of $\mathrm{MoS}_{5} \mathrm{Si}_{3}, \mathrm{P}$. F. Tortorelli, B. A. Pint, K. L. More, A. J. Thom, and M. Akinc

Modeling of Chemical Vapor Deposited Zirconia for Thermal Barrier and Environmental Barrier Coatings, T. Starr, W. Xu, T. R. Barnett, T. M. Besmann, and V. Veranasi

\section{Advanced Conventional Alloys}

Evaluation of Stainless Steels for Primary Surface Recuperator Applications, R. W. Swindeman, P. J. Maziasz, B. A. Pint, J. P. Montague, and M. Fitzpatrick

\section{ODS Alloys}

Fireside Corrosion Testing of Candidate Superheater Tube Alloys, Coatings, and Claddings-Phase III, J. L. Blough and W. W. Seitz

\section{Ultrahigh Performance Materials Poster Presentations}

Investigation of the Cr-Ta Alloy Composites Reinforced by the Cr $r_{2}$ Ta Laves Phase, Y. H. He, D. F. Wang, P. Liaw, C. T. Liu, L. Heatherly, and E. P. George

Computational Hydrogen Embrittlement and Thermal Expansion Simulations of Iron Aluminides, R. Cisloiu, B. S.-J. Kang, and B. R. Cooper 


\section{FINAL PROGRAM \\ $15^{\text {th }}$ ANNUAL CONFERENCE ON FOSSIL ENERGY MATERIALS \\ Hyatt Regency Hotel \\ Knoxville, Tennessee \\ April 30-May 2, 2001}

\section{Monday, April 30}

Registration 12:00-1:00 P.M.

1:00 Welcome and Introductory Remarks, R. R. Judkins, Oak Ridge National Laboratory

Session I-Functional Materials

1:10 Introductory Remarks, T. R. Armstrong, Oak Ridge National Laboratory

1:20 Invited Presentation-Ted McMahon, National Energy Technology Laboratory: The NETL Gas Separations Program

2:00 Efficient Production of Pure Hydrogen from Hydrocarbons Using Palladium Membrane Reactors, S. A. Birdsell, Los Alamos National Laboratory

2:25 A Multiprogram Initiative to Develop Hydrogen Separation and Purification Membranes, D. Loy, Sandia National Laboratories

2:50 Development of Inorganic Membranes for Gas Separation, D. E. Fain, East Tennessee Technology Park

\section{3:15 Break}

3:45 Bismuth Oxide Solid Electrolyte Oxygen Separation Membranes, S. D. Nunn, Oak Ridge National Laboratory
4:10 Solid Oxide Electrolyte Systems,

L. Pederson, Pacific Northwest National Laboratory

4:35 Development of Novel Activated Carbon Composites, T. D. Burchell, Oak Ridge National Laboratory

5:00 Adjourn

5:30 Invited Presentation-Update on the Vision 21 Program, L. Ruth, National Energy Technology Laboratory

5:55 Reception and Poster Session

\section{Tuesday, May 1}

7:00 Continental Breakfast

\section{Session II-Coatings and Protection of Materials}

8:00 Introductory Remarks

8:10 Invited Presentation, S. Osgerby, NPL, Towards Lifetime Prediction Models for Gas Turbine Coatings-Past, Present and Future Work at NPL

8:40 Investigation of Iron Aluminide Weld Overlays, J. N. DuPont, Lehigh University

9:05 Coating Microstructure-Property Issues, R. N. Wright, Idaho National Engineering and Environmental Laboratory

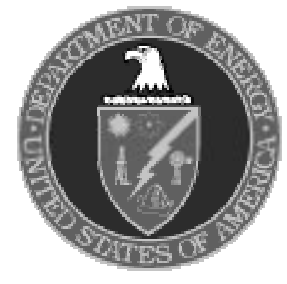

SPONSORED BY THE UNITED STATES DEPARTMENT OF ENERGY, OFFICE OF FOSSIL ENERGY,

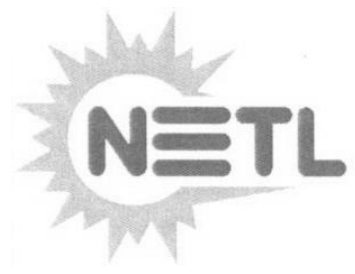


9:30 CVD Metallic Coatings/Extended

Lifetime Coatings, B. A. Pint, Oak

Ridge National Laboratory

9:55 Break

10:25 Slurry-Based Mullite Coatings for Corrosion Resistance, B. L. Armstrong, Oak Ridge National Laboratory

10.45 Development of Nondestructive Evaluation Methods for Ceramic Coatings, W. A. Ellingson, Argonne National Laboratory

11:05 Chemically Vapor Deposited YSZ for Thermal and Environmental Barrier Coatings, T. M. Besmann, J. C. McLaughlin Oak Ridge National Laboratory and V. Varanasi, University of Florida

11:25 High-Temperature Materials Testing in a Pilot-Scale Coal Combustion System, J. Hurley and C. R. Crocker, University of North Dakota, Energy and Environmental Research Center

11:45 Lunch

\section{Session III-New Alloys}

1:30 Introductory Remarks, I. G. Wright, Oak Ridge National Laboratory

1:35 Invited Presentation, Overview of Materials Needs for Advanced Steam Generators, R. Viswanathan, EPRI

2:05 Progress on Developing Codes and Standards Requirements for New Alloys, R. W. Swindeman, Oak Ridge National Laboratory

2:30 ODS Alloy Development, I. G. Wright, Oak Ridge National Laboratory
2:55 Reduction in Defect Content in ODS Alloys, A. R. Jones, University of Liverpool

3:20 Break

3:45 Optimization of ODS Alloy Properties, B. Kad, University of California at San Diego

4:10 Corrosion and Mechanical Properties of Materials in Combustion and MixedGas Environments, K. Natesan, Argonne National Laboratory

4:35 High Creep-Strength Alloys, P. J. Maziasz, Oak Ridge National Laboratory (CRADA with INCO)

5:00 Adjourn

\section{Wednesday, May 2}

7:00 Continental Breakfast

\section{Session IV-Albany Research Center- Advanced Metallurgical Processes Program}

8:00 Introductory Remarks

8:10 Overview: Fossil Energy Advanced Metallurgical Processes Program, R. P. Walters, Albany Research Center

8:20 Oxidation/Sulfidation Resistant Materials, J. S. Dunning, Albany Research Center

8:45 Oxidation in a Temperature Gradient, G. R. Holcomb, Albany Research Center

9:10 Erosive Wear of Selected Materials for Fossil Energy Applications, J. C. Rawers, Albany Research Center

\author{
SPONSORED BY THE UNITED STATES \\ DEPARTMENT OF ENERGY, \\ OFFICE OF FOSSIL ENERGY,


9:35 Break

10:05 Advanced Titanium Processing, A. D. Hartman, Albany Research Center

10:30 Microchannel Devices, D. E. Alman, Albany Research Center

10:55 Refractory Failure in IGCC Fossil Fuel Power Systems, C. P. Dogan, Albany Research Center

11:20 Lunch

\section{Session V-Ultrahigh Performance Materials}

1:00 Introductory Remarks, D. P. Stinton, Oak Ridge National Laboratory

1:15 Multi-Phase Cr-Based Alloys for Aggressive High Temperature Environments, M. P. Brady, Oak Ridge National Laboratory

1:40 Mo-Si Alloy Development, J. H. Schneibel, Oak Ridge National Laboratory

2:05 Mo-Si Alloy Development, M. J. Kramer, Ames Laboratory

2:30 Break

3:00 Development of a Commercial Process for the Production of Silicon Carbide Fibrils, R. D. Nixdorf, ReMaxCo Technologies, Inc

3:25 Closing remarks and adjourn

\section{Posters}

\section{Fuel Cells}

Fabrication Technology for Fuel Cell Containment and Liners, M. L. Santella, Oak Ridge National Laboratory
Improved Fuel Cell Materials and Economical Fabrication, T. R. Armstrong, Oak Ridge National Laboratory

Performance Evaluation of Thin-Film Multi-

Layer Packages, K. Reifsnider, Virginia Polytechnic Institute

Low Cost Processing Technique For Solid Oxide Fuel Cells, U. B. Pal and V. K. Sarin, Boston University

\section{Gas Cleaning/Separation}

Processing Analysis and Characterization of Membranes for Hydrogen Separation, T. R.

Armstrong, Oak Ridge National Laboratory

Bismuth Oxide Solid Electrolyte Systems, L. Pederson, Pacific Northwest National Laboratory

\section{Gas Cleaning}

Metallic Filters for Hot Gas Cleaning, I. E. Anderson, Ames Laboratory

\section{Coatings and Protection of Materials}

High Temperature Materials Testing in Coal Combustion Environments, M. Mathur and M. Freeman, National Energy Technology Laboratory

Reliable Ceramic Coatings for HighTemperature Environmental Resistance in Fossil Environments, P. F. Tortorelli, Oak Ridge

National Laboratory

Modeling of Chemically Vapor Deposited Zirconia for Thermal Barrier and Environmental Barrier Coatings, T. Starr, University of Louisville

\section{Advanced Conventional Alloys} Improved Recuperator Alloys, P. J. Maziasz, Oak Ridge National Laboratory (CRADA with Solar Turbines) 


\section{ODS Alloys}

In-Plant Corrosion Probe Tests, J. L. Blough, Foster Wheeler Development Corporation

Development of MA956 Tubes (joint with Special Metals), I. G. Wright, Oak Ridge National Laboratory
Ultrahigh Performance Materials Poster Presentations

Study of Fatigue and Fracture Behavior of $\mathrm{Cr}$ Based Alloys and Intermetallic Materials, P. Liaw, University of Tennessee

Evaluation of the Intrinsic and Extrinsic Fracture Behavior of Iron Aluminides, B. R. Cooper, West Virginia University

SPONSORED BY THE UNITED STATES

DEPARTMENT OF ENERGY,

OFFICE OF FOSSIL ENERGY, 


\section{Fossil Energy Materials Conference \\ Knoxville, Tennessee \\ April 30-May 2, 2001}

\section{Attendee List}
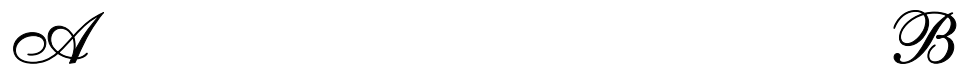

David Alman

Albany Research Center

U.S. DOE

1450 Queen Ave. SW

Albany, OR 97321

Phone: 541-967-5885

Fax: 541-967-5845

Email: alman@alrc.doe.gov

\section{Mary Anne Alvin}

Siemens Westinghouse Power Corporation

Science and Technology Center

1310 Beulah Road

Pittsburgh, PA 15235

Phone: 412-256-2066

Fax: 412-256-2121

Email: maryanne.Alvin@swpc.siemens.com

Iver E. Anderson

Ames Laboratory

124 Metals Development Building

Ames, IA 5001

Phone: 515-294-4446

Fax: 515-294-8727

Email: andersoni@ameslab.gov

\section{Tim Armstrong}

Oak Ridge National Laboratory

P.O. Box 2008

Oak Ridge, TN 37831-6084

Phone: 865-574-7996

Fax: 865-574-4357

Email: armstrongt@ornl.gov

\section{Sarah K. Baham}

University of Tennessee

Department of Materials Science and Engineering

434 Dougherty Engineering Building

Knoxville, TN 37996

Phone: 865-591-9495

Fax: 865-974-4115

Email:sbaham@utk.edu

Brian A. Baker

Special Metals Corporation

3200 Riverside Drive

Huntington, WV 25705

Phone: 304-526-5740

Fax: 304-526-5973

Email: bbaker@smewv.com

\section{Stephen Balsone}

GE Power Systems

1 River Road, Bldg 55-119

Schenectady, NY 12345

Phone: 518-387-0466

Fax: 518 387-4983

Email: stephen.balsone@ps.ge.com

\section{Theodore M. Besmann}

Oak Ridge National Laboratory

P.O. Box 2008

Oak Ridge, TN 37831-6063

Phone: 865-574-6852

Fax: 865-574-6918

Email: besmanntm@ornl.gov 


\author{
Jerry L. Bitner \\ JLB Engineering, Inc. \\ 5979 Bethel Park Drive \\ Bethel Park, PA 15102 \\ Phone: 412-854-1427 \\ Fax: 412-854-1427 \\ Email: Jerry_Bitner@msn.com \\ J. L. Blough \\ Foster Wheeler Development Corporation \\ Phone: 973-535-2355 \\ Fax: 973-535-2242 \\ Email: Jeff_Blough@fwc.com \\ Craig A. Blue \\ Oak Ridge National Laboratory \\ P.O. Box 2008 \\ Oak Ridge, TN 37831-6083 \\ Phone: 865-574-4351 \\ Fax: 865-574-4357 \\ Email: blueca@ornl.gov \\ Mike Brady \\ Oak Ridge National Laboratory \\ P.O. Box 2008 \\ Oak Ridge, TN 37831-6115 \\ Phone: 865-574-5153 \\ Fax: 865-574-7659 \\ Email: bradymp@ornl.gov \\ Timothy D. Burchell \\ Oak Ridge National Laboratory \\ P.O. Box 2008 \\ Oak Ridge, TN 37831-6088 \\ Phone: 865-576-8595 \\ Fax: 865-576-8424 \\ Email: burchelltd@ornl.gov
}

J. David Carter

Argonne National Laboratory

CMT Blg 205/A-159

9700 So. Cass Avenue

Argonne, Illinois, 60439

Phone: 630-252-4544

Fax: 630-972-4544

Email: carter@cmt.anl.gov

Nancy C. Cole

NCC Engineering

2735 Robert Oliver Ave.

Fernandina Beach, FL 32034

Phone: 904-491-5089

Fax: 904-491-5089

Email: nccengr@net-magic.net

Kevin Cooley

Oak Ridge National Laboratory

1 Bethel Valley Road, MS 6063

Oak Ridge, TN 37831-6063

Phone: 865-574-4559

Fax: 865-574-6918

Email: cooleykm@ornl.gov

Bernard S. Covino, Jr.

Albany Research Center

U.S. DOE

1450 Queen Ave. SW

Albany, OR 97321

Phone: 541-967-5828

Fax: 541-967-5914

Email: covino@alrc.doe.gov

\section{Michael Cybulsky}

Alstom Power Inc.

2000 Day Hill Rd.

Windsor, CT 06095

Phone: 860-285-3717

Fax: 860-285-2513

Email: Michael.Cybulsky@power.alstom.com 


\section{Cindy P. Dogan}

U.S. Department of Energy

Albany Research Center

1450 Queen Ave., S. W.

Albany, OR 97321

Phone: 541-967-5803

Fax: 541-967-5845

Email: dogan@alrc.doe.gov

\section{John Dunning}

Albany Research Center

U.S. DOE

1450 Queen Ave. SW

Albany, OR 97321

Phone: 541-967-5876

Fax: 541-967-5845

Email: dunning@alrc.doe.gov

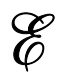

William A. (Bill) Ellingson

Argonne National Laboratory

9700 South Cass Avenue

Argonne, IL 60439-4838

Phone: 630-252-5068

Fax: 630-252-4798

Email: ellingson@anl.gov

Bob Enick

Chemical Engineering

1249 Benedum Hall

University of Pittsburgh

Pittsburgh, PA 15261

Phone: 412-624-9649

Fax: 412-624-9639

Email: enick@engrug.pitt.edu

\section{Douglas E. Fain}

Bechtel Jacobs Company LLC

Inorganic Membrane Technology Laboratory

P.O. Box 4699, K-1037, MS-7271

East Tennessee Technology Park

Oak Ridge, Tennessee 37831

Phone: 865-574-9932

Fax: 865-576-2930

E-Mail fainde@bechteljacobs.org

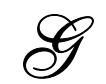

\section{Fred Glaser}

Department of Energy 19901 Germantown Rd.

Germantown, MD 20874

Phone: 301-903-2676

Fax: 301-903-0243

Email: Fred.Glaser@hq.doe.gov

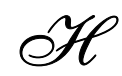

\section{Mark A. Harper}

Special Metals Corporation

3200 Riverside Drive

Huntington, WV 25705

Phone: 304-526-5057

Fax: 304 526-5973

Email: mharper@smcwv.com

\author{
Alan D. Hartman \\ Albany Research Center \\ DOE \\ 1450 Queen Ave. SW \\ Albany, OR 97321 \\ Phone: 541-967-5862 \\ Fax: 541-967-5958 \\ Email: Hartman@alrc.doe.gov
}


Jeffrey Hawk

Albany Research Center

U.S. DOE

1450 Queen Ave. S.W.

Albany, OR 97321

Phone: 541-967-5900

Fax: 541-967-5845

Email: hawk@alrc.doe.gov

\section{Richard Higgins}

CeraMem Corporation

12 Clematis Ave.

Waltham, MA 02453

Phone: 781-899-4495 Ex. 23

Fax: 781-899-6478

Email: Higgins@ ceramem.com

\section{Deidre Hirshfeld}

Fluore Science Inc.

819 Zia Lane

Espanola, NM 87532

Or P.O. Box 981

Socorro, NM 87801

Phone: 505-747-3338

505-835-5129

Fax: 505-753-6863

505-835-5626

Email: Hirsch@nmt.edu

\section{Gordon Holcomb}

Albany Research Center

U.S. DOE

1450 Queen Ave. SW

Albany, OR 97321

Phone: 541-967-5874

Fax: 541-967-5914

Email: holcomb@alrc.doe.gov

\section{Xinyu Huang}

Virginia Polytechnic

Morgantown, WV 26506

Phone: 540-231-3139

Fax: 540-231-9187

Email: xihuang@vt.edu

\section{John Hurley}

Energy \& Environmental Research Center

P.O. Box 9018

Grand Forks, ND 58202-9018

Phone: 701-777-5159

Fax: 701-777-5181

Email: jhurley@undeerc.org

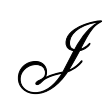

\section{Andy Jones}

University of Liverpool

Faculty of Engineering, 3 Brownlow St., Liverpool, L69 3GL, UK

Phone: No. +44 1517948026

Fax: +44 1517948070

Email: a.r.jones@liv.ac.uk

\section{Roddie R. Judkins}

Oak Ridge National Laboratory

P.O. Box 2008

Oak Ridge, TN 37831-6084

Phone: 865-574-4572

Fax: 865-574-4357

Email: judkinsrr@ornl.gov

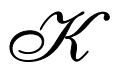

\section{Bimal Kad}

University of California at San Diego

Address

Phone: 858-534-7059

Fax: 858-534-6373

Email: BKAD@ucsd.edu

\section{Bruce Kang}

West Virginia University

353 ESB, Mech. \& Aerospace Engr. Dept.

Morgantown, WV 26506

Phone: 304-293-3111 ext. 2361

Fax: 304-293-6689

Email: BSKang@mail.wvu.edu 


\section{Art Kracke}

Allvac

2020 Ashcraft Avenue

Monroe, NC 28110

Phone: 704-225-7353

Fax: 704-226-0446

Email: art.kracke@allvac.com

Matt J. Kramer

Ames Laboratory

37 Wilhelm Hall

Ames, IA 5001

Phone: 515-294-0276

Fax: 515-294-4291

Email: mjkramer@ameslab.gov

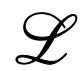

\section{Edgar Lara-Curzio}

Oak Ridge National Laboratory

1 Bethel Valley Rd. BLDG. 4515, MS-6069

Oak Ridge, TN 37831-6069

Phone: 865-574-1749

Fax: 865-574-6098

Email: laracurzioe@ornl.gov

\section{Peter K. Liaw}

The University of Tennessee

Dept. of Materials Science and Engineering

Knoxville, TN 37996-2200

Phone: 865-974-6356

Fax: 865-974-4115

Email: liaw@utkux1.utk.edu

\section{Douglas A. Loy}

Sandia National Laboratories

87185-0888

Phone: 505-844-4445

Fax: 505084409624

Email: daloy@sandia.gov
Arnold R. Marder

Lehigh University

Materials Science and Engineering

Whitaker Laboratory

Bethlehem, PA 18015

Phone: 610-758-4197

Fax: 610-758-6407

Email: arm0@Lehigh.EDU

\section{Bruce Marshall}

Inorganic Membrane Technology Laboratory

Bechtel Jacobs Company, LLC, P.O. Box 4699

Oak Ridge, TN 37831-7271

Phone: 865-574-0879

Fax: 865-576-2930

Email: bbm@bechteljacobs.org

\section{Mahendra (Matt) Mathur}

National Energy Technology Laboratory

U.S. Department of Energy

P.O. Box 10940

Pittsburgh, PA 15236

Phone: 412-386-4605

Fax: 412-386-6004

Email: Mahendra.Mathur@netl.doe.gov

\section{Phil Maziasz}

Oak Ridge National Laboratory

P.O. Box 2008

Oak Ridge, TN 37831-6115

Phone: 865-574-5082

Fax: 865-574-7659

Email: maziaszpj@ornl.gov

Claudette McKamey

Oak Ridge National Laboratory

P.O. Box 2008

Oak Ridge, TN 37831-6115

Phone: 865-574-6917

Fax: 865-574-7659

Email: mckameycg@ornl.gov 


\author{
Ted McMahon \\ PM-60 \\ National Energy Technology Laboratory \\ U.S. Department of Energy \\ 3610 Collins Ferry Road \\ P.O. Box 880, Mail Stop C04 \\ Morgantown, WV 26507-0880 \\ OfficeB26-266 \\ Mailstop: $\mathrm{C} 04$ \\ Phone: 304-285-4865 \\ Fax: 304-285-4403 \\ Email: ted.mcmahon@netl.doe.gov

\section{Ravi Menon} \\ Thermadyne-Stoody \\ 5557 Nashville Road \\ Bowling Green, KY 42101 \\ Phone: 270-781-9777 ext. 2009 \\ Fax: 270-782-9237 \\ Email: ravi_menon@thermadyne.com

\section{Walt Mohn} \\ McDermott Technology, Inc. \\ 1562 Beeson Street \\ Alliance, Ohio 44601 \\ Phone: 330-829-7816 \\ Fax: 330-829-7381 \\ Email: walter.r.Mohn@mcdermott.com
}

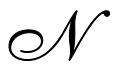

\section{Ken Nateson}

Argonne National Laboratory

Phone: 630-252-5103

Fax: 630-972-4544

Email: nateson@anl.gov

\section{Birendra Nath}

Innogy plc

Windmill Hill Business Park

Whitehill Way

Swindon (UK)

SN5 6PB

Phone: +44-(0)1793-89-6288

Fax: +44-(0)1793-89-2288

Mobile: +44-(0)798-949-3845

Email: BIRENDRA.NATH@innogy.com

\section{Dick Nixdorf}

ReMaxCo Technologies, Inc.

1010 Commerce Park Drive,Suite I

Oak Ridge, TN 37830

Phone: 865-482-7552 ext. \#1

Fax: 865-482-7505

Email: nixdorfr@indceramicsolns.com

\section{Darryl Noreen}

ZeTek Power

717 Fifth Ave., $4^{\text {th }}$ Floor

New York, NY 10022

Phone: 212-758-8095

Fax: 212-758-8209

Email: darrylnoreen@zetekpower.com

\section{Stephen D. Nunn}

Oak Ridge National Laboratory

P.O. Box 2008

Oak Ridge, TN 37831-6087

Phone: 865-576-1668

Fax: 865-574-8271

Email: nunnsd@ornl.gov

\section{(0)}

\section{John Oakey}

Cranfield University

Power Generation Technology Centre

Cranfield

Bedford, MK43 0Al

Phone: +44(0)1234 754253

Fax: +44(0)1234 752473

Email: j.e.oakey@cranfield.ac.uk

Steve Osgerby

National Physical Laboratory

Teddington, UK

Middlesex TW11 OLW

Phone: 442089436521

Fax: 442089436177

Steve.osgerby@npl.co.uk 


\section{$\mathscr{P}$}

\section{Steve Paglieri}

Los Alamos National Laboratory

P.O. Box 1663, MS-C348

Los Alamos, NM 87545

Phone: 505-667-1669

Fax: 505-665-1687

Email: steve.paglieri@lanl.gov

\section{Arvid Pasto}

Oak Ridge National Laboratory

High Temperature Materials Laboratory

P.O. Box 2008

Oak Ridge, TN 37831-6062

Phone: 865-574-5123

Fax: 865-574-4913

Email: pastoae@ornl.gov

E. Andrew Payzant

Oak Ridge National Laboratory

P.O. Box 2008

Oak Ridge, TN 37831-6064

Phone: 865-574-6538

Fax: 865-574-3940

Email: payzanta@ornl.gov

\section{Larry Pederson}

Pacific Northwest National Laboratory

Phone: 509-375-2771

Fax: 509-375-2167

Email: Larry.Pederson@pnl.gov

\section{Bruce A. Pint}

P.O. Box 2008

Oak Ridge, TN 37831-6156

Phone: 865-576-2897

Fax: 865-241-0215

Email: pintba@ornl.gov

\section{$\mathscr{R}$}

\section{Udaya Rao}

NETL

P.O. Box 10940

Pittsburgh, PA 15236-0940

Phone: 412-386-4743

Fax: 412-386-5917

Email: Rao@netl.doe.gov

\section{Mary H. Rawlins}

U.S. Department of Energy

P.O. Box 2001

Oak Ridge, TN 37831

Phone: 865-576-4507

Fax: 865-576-0392

Email: rawlinsmh@ornl.gov

Richard B. Read

Office of Project Management

National Energy Technology Laboratory

P.O. Box 10940

Pittsburgh, PA 15236-0940

Phone: 412-386-5721

Fax: 412-386-5917

Email: read@netl.doe.gov

\section{K. Reifsnider}

Virginia Polytechnic Institute

Assoc Prov Interdis Prog

Materials Response Group/ESM

Blacksburg, VA 24061

Phone: 540-231-5316

Fax: 540-231-9187

Email: mrl@vt.edu

Billie Jean Russell

Oak Ridge National Laboratory

High Temperature Materials Laboratory

P.O. Box 2008

Oak Ridge, TN 37831-6062

Phone: 865-574-1926

Fax: 865-574-4913

Email: russellbj@ornl.gov

\section{Lawrence A. Ruth}

CE-01

U.S. Department of Energy

National Energy Technology Laboratory

P.O. Box 10940

Pittsburgh, PA 15236-0940

Mailstop: 922-204

Phone: 412-386-4461

Fax: 412-386-4822

Email: ruth@netl.doe.gov 


\author{
Michael Rutkowski \\ Parsons \\ 1 Meridian Boulevard, Suite 2B-1 \\ Wyomissing, Pennslyvania 19610 \\ Phone: 484-338-2292 \\ Fax: 484-338-2354 \\ Email: Michael.D.Rutkowski@Parsons.com
}

\section{Mike Santella}

Oak Ridge National Laboratory

P.O. Box 2008

Oak Ridge, TN 37831-6096

Phone: 865-574-4805

Fax: 865-574-4928

Email: santellaml@ornl.gov

Vinod K. Sarin

College of Engineering

Boston University

15 St. Mary's Steet

Boston, MA 02215

Phone: 617-353 6451

Fax: 617-353 5548

Email: Sarin@engc.bu.edu

\section{Joachim H. Schneibel}

Oak Ridge National Laboratory

P.O. Box 2008

Oak Ridge, TN 37831-6115

Phone: 865-576-4644

Fax: 865-574-7659

Email: schneibeljh@ornl.gov

\author{
Marvin I. Singer \\ Director, Office of Advance Research \\ FE-25 \\ Forrestal Building \\ Room 4G-052 \\ Washington, DC \\ Phone: 202-586-4336 \\ Fax: 202-586-9352 \\ Email: marvin.singer@hq.doe.gov
}

Gaylord D. Smith

Special Metals Corporation

3200 Riverside Drive

Huntington, WV 25705

Phone: 304-526-5735

Fax: 304-526-5735

Email: gsmith@smewv.com

\section{Thomas L. Starr}

Chemical Engineering Department

University of Louisville

Louisville, KY 40292

Phone: 502-852-1073

Fax: 502-852-6355

E-mail tom.starr@louisville.edu

\section{Dave Stinton}

Oak Ridge National Laboratory

P.O. Box 2008

Oak Ridge, TN 37831-6084

Phone: 865-574-4556

Fax: 865-574-6918

Email: stintondp@ornl.gov

\section{Robert W. Swindeman}

Oak Ridge National Laboratory

P.O. Box 2008

Oak Ridge, TN 37831-6155

Phone: 865-574-5108

Fax: 865-574-5118

Email: swindemanrw@ornl.gov

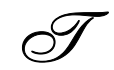

Peter F. Tortorelli

P.O. Box 2008

Oak Ridge, TN 37831-6156

Phone: 865-574-5119

Fax: 865-241-0215

Email: tortorellipf@ornl.gov 


\section{$\mathscr{Q}$}

Venu G. Varanasi

Oak Ridge National Laboratory

P.O. Box 2008

Oak Ridge, TN 37831-6063

Phone: 865-241-4537

Fax: 865-574-6918

Email: varanasiv@ornl.gov

\section{R. Viswanathan}

Electric Power Research Institute 3412 Millview Ave.

Palo Alto, CA 94350

Phone: 650-855-2450

Fax: 650-855-1026

Email: R.viswana@epri.com

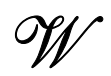

\section{Rich Walters}

Albany Research Center

U.S. DOE

1450 Queen Ave. S.W.

Albany, OR 97321

Phone: 541-967-5873

Fax: 541-967-5991

Email: walters@alrc.doe.gov

\section{Diafeng Wang}

The University of Tennessee

Dept. of Materials Science and Engineering

Knoxville, TN 37996-2200

Phone: 865-974-0645

Fax: 865-974-4115

Email: dwang@utk.edu

Ian G. Wright

Oak Ridge National Laboratory

P.O. Box 2008

Oak Ridge, TN 37831-6156

Phone: 865-574-4451

Fax: 865-241-0215

Email: wrightig@ornl.gov

\section{Richard N. Wright}

Idaho National Engineering and Environmental Lab

PO Box 1625 MS2218

Idaho Falls, ID 83415-2218

Phone: 208-526-6127

Fax: 208-526-0690

Email: RNW2@INEL.gov 